\title{
TIME DOMAIN RESPONSE OF UNIFORM RC LINES WITH RESISTIVE TERMINATION AT BOTH ENDS
}

\author{
MUHAMMAD TAHER ABUELMA'ATTI \\ Kung Fahd University of Petroleum and Minerals, Box 203, Dhahran 31261, Saudi Arabia \\ (Received October 30, 1993; in final form December 12, 1993)
}

The time domain response of uniform RC lines with resistive termination at both ends is obtained by obtaining the approximate poles of the transfer function. A simple model is developed for the URC line. The model consists of three passive elements only and can be easily implemented for computeraided analysis of URC lines with resistive termination at both ends.

\section{INTRODUCTION}

Uniformly distributed (URC) lines are widely used in many diverse fields, for example for modelling of VLSI interconnects. The analytical solutions for timedomain response of open-circuit, short-circuit, capacitively loaded, infinitely long, and capacitively-resistively loaded URC lines have received considerable attention in the literature [1-12]. In all these cases it is assumed that the URC line is driven by an ideal voltage source with zero source resistance. This is not the case in practical situations where the URC line is usually driven by a source with a finite resistance. Thus, the case of a URC line with resistive termination at both ends is of practical importance. Nevertheless, it has received very little attention in the literature. This is probably attributed to the difficulty of obtaining the exact, or even the approximate poles of the mathematically untractable transfer function of this case. In an attempt to find an approximate analytical solution for this case, Milic and Gmitrovic [13] presented a technique based on Liou's [14] procedure, which obviates the finding of the poles of the transfer function. This paper presents an alternative analytical solution based on finding the approximate poles of the transfer function of the URC line with resistive termination at both ends, over a wide range of load, source, and line parameters. Based on this analytical solution, a simple equivalent circuit, formed by three passive elements only, is derived for the URC line with resistive termination at both ends. This model is compatible with previously published models and would be more appropriate for computeraided analysis of circuits and systems comprising URC lines with resistive termination at both ends. 


\section{ANALYSIS}

The transfer function of a voltage driven (Fig. 1) URC line with resistive termination at both ends can be expressed by

$$
\begin{aligned}
& \frac{V_{L}(s)}{V_{i}(s)} \\
& \quad=\frac{1}{\left(\left(1+R_{S} / R_{L}\right) \cosh \sqrt{s C R}+\left(\left(R / R_{L}\right) / \sqrt{s C R}+\left(R / R_{S}\right) \sqrt{s C R}\right) \sinh \sqrt{s C R}\right)}
\end{aligned}
$$

where $R_{S}$ is the source resistance, $R_{L}$ is the load resistance, $R$ is the total resistance, and $\mathrm{C}$ is the total capacitance of the line. Denoting $\mathrm{sCR}=\psi, \alpha=\mathrm{R}_{\mathrm{S}} / \mathrm{R}$ and $\beta=$ $R / R_{L}$, eqn (1) reduces to

$\frac{\mathrm{V}_{\mathrm{L}}(\psi)}{\mathrm{V}_{\mathrm{i}}(\psi)}=\frac{1}{(1+\alpha \beta) \cosh \sqrt{\psi}+(\beta / \sqrt{\psi}+\alpha \sqrt{\psi}) \sinh \sqrt{\psi}}=\frac{1}{\mathrm{Q}(\alpha, \beta, \psi)}$

The transfer function of an open-circuit URC line can be obtained from eqn. (2) by setting $\beta=0$.

Fig. 2 shows a plot of $\mathrm{Q}(\alpha, \beta, \psi)$ for different values of $\alpha, \beta$, and $\psi$. Inspection of Fig. 2 reveals that, in general, $\mathrm{Q}(\alpha, \beta, \psi)$ can be represented by polynomials of the Nth order of the form

$\mathrm{Q}(\alpha, \beta, \psi)=\gamma_{\mathrm{o}}+\sum_{\mathrm{n}=1}^{\mathrm{N}} \gamma_{\mathrm{n}} \psi^{\mathrm{n}}$

where $\gamma_{\mathrm{o}}=1+\beta(1+\alpha)$. In general, the parameters $\gamma_{\mathrm{n}}$ can be obtained by using standard curve-fitting subroutines available in most mainframe computers. However, for a second-order polynomial, with $\mathbf{N}=2$, the parameters $\gamma_{1}$ and $\gamma_{2}$ can be obtained by hand calculations using the Lagrange interpolating polynomial [15]. This procedure yields a family of parameters $\gamma_{1}$ and $\gamma_{2}$ that depend on $\alpha$ and $\beta$.

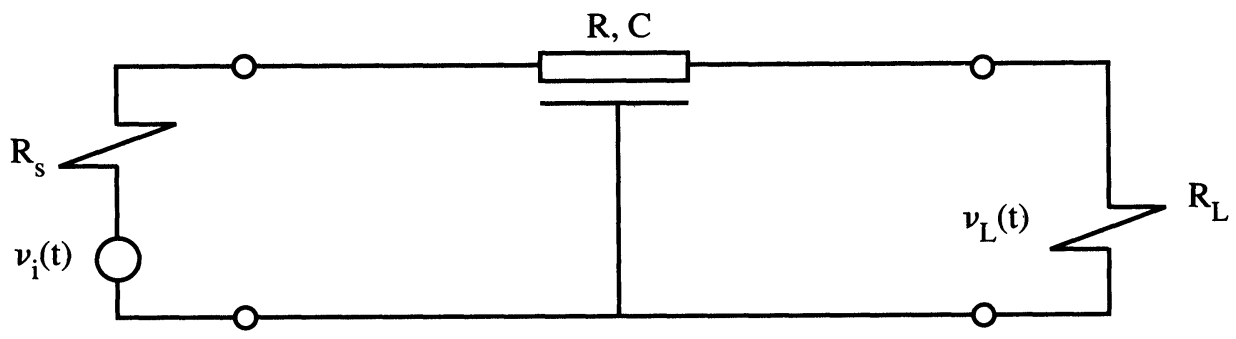

FIGURE 1 Voltage-driven URC line with resistive termination at both ends. 


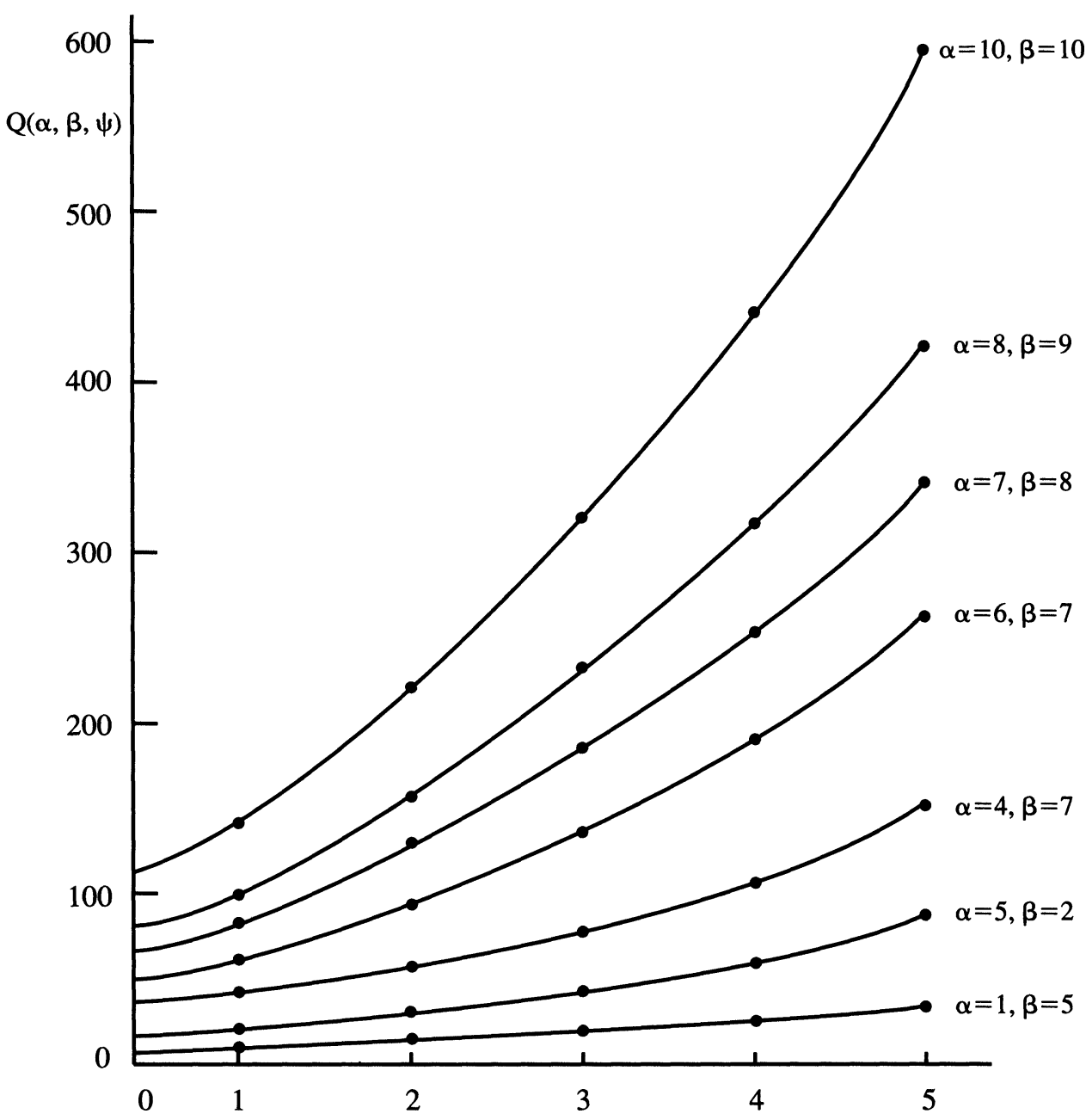

FIGURE 2 Variation òf $\mathrm{Q}(\alpha, \beta, \psi)$ with the parameters $\alpha$ and $\beta$ calculated with $(2)(-)$ and calculated with $(3-5)(\bullet)$.

These parameters are fitted to simple closed-form analytical expressions, giving

$$
\begin{aligned}
& \gamma_{1}=0.4745+0.91 \alpha+(0.166+0.4822 \alpha) \beta \\
& \gamma_{2}=0.0555+0.2718 \alpha+(0.01085+0.05793 \alpha) \beta
\end{aligned}
$$

Using eqn. (3)-(5), calculations were made with $\mathrm{N}=2$, and are shown in Fig. 2. It is seen that the proposed second-order polynomial of eqn. (3) accurately represents the denominator of eqn. (1) with an average root-mean-square error contained within $0.95 \%$ for $0<\alpha<10$ and $0<\beta<10$. 


\section{RESULTS}

By combining eqn. (3)-(5) and substituting into eqn. (2), assuming $N=2$, the unit step response due to unput $V_{i}(t)=u(t)$ is

$\mathrm{V}_{\mathrm{L}}(\mathrm{s})=\frac{1}{\mathrm{~s}} \frac{1}{\gamma_{\mathrm{o}}+\gamma_{1} \psi+\gamma_{2} \psi^{2}}$

or

$\frac{\mathrm{V}_{\mathrm{L}}(\psi)}{\mathrm{RC}}=\frac{1}{\gamma_{2}} \frac{1}{\psi^{2}+2 \mathrm{~b} \psi+\omega^{2}}$

where $2 b=\gamma_{1} / \gamma_{2}$ and $\omega_{0}^{2}=\gamma_{0} / \gamma_{2}$. Since $\omega_{0}^{2}<b^{2}$, the inverse Laplace transform of eqn. (6) is obtained as [15]

$\mathrm{V}_{\mathrm{L}}(\mathrm{t})=\left(1-\frac{\omega_{\mathrm{o}}^{2}}{\mathrm{p}-\mathrm{m}}\left(\frac{\mathrm{e}^{\mathrm{mt} / \mathrm{RC}}}{\mathrm{m}}-\frac{\mathrm{e}^{\mathrm{pt} / \mathrm{RC}}}{\mathrm{p}}\right)\right)$

where $\mathrm{m}$ and $\mathrm{p}$ are the roots of the equation

$\psi^{2}+2 b \psi+\omega_{0}^{2}=0$

Fig. 3 depicts the unit-step response for the URC line with resistive termination at both ends for different values of source and load resistances. From Fig. 3, it is

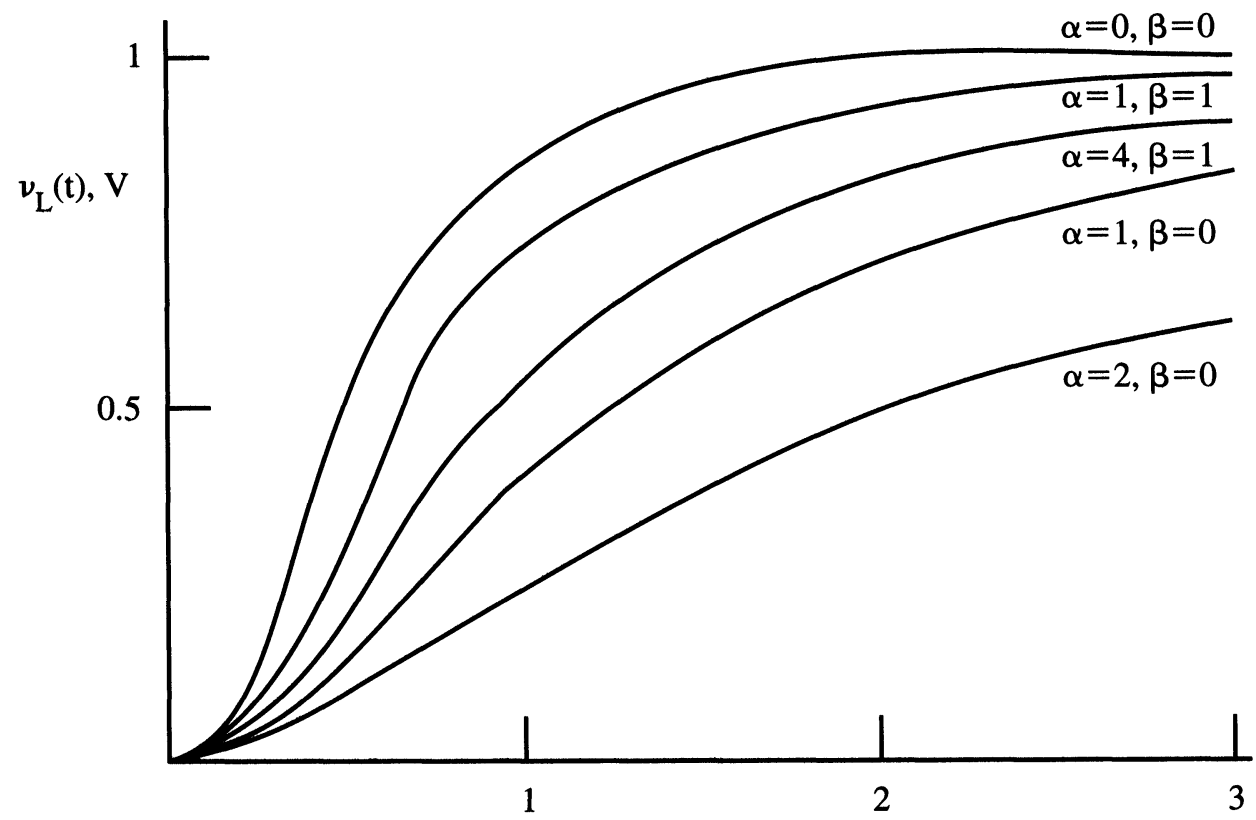

FIGURE 3 Unit-step response for URC with resistive termination at both ends. ——: Calculated (7) - - - exact. 


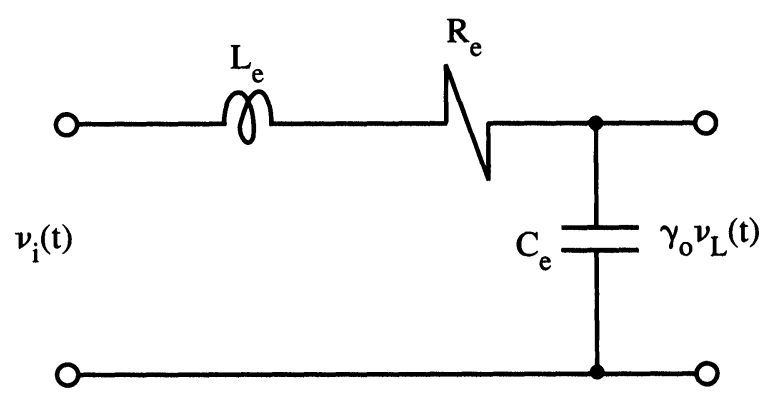

FIGURE 4 Proposed model for URC line with resistive termination at both ends. $\mathrm{Re}=\mathrm{R}, \mathrm{Ce}=$ $\gamma_{1} / \gamma_{1} \mathrm{C}, \mathrm{Le}=\gamma_{2} / \gamma_{\mathrm{v}} \mathrm{CR}$, and $\gamma_{0}=1+\beta(1+\alpha), \gamma_{1}$ and $\gamma_{2}$ given by eqns. (4) and (5), respectively.

obvious that the approximations of eqn. (3)-(5) accurately predict the performance of the URC line resistively terminated at both ends.

From eqn. (2)-(5) it can be shown that the transfer function of the URC line resistively terminated at both ends corresponds to the equivalent circuit shown in Fig. 4. This equivalent circuit consists of three passive elements only and is, therefore, appropriate for computer-aided design of circuits and systems comprising URC lines with resistive termination at both ends.

\section{CONCLUSION}

In this paper, a second-order polynomial representation has been proposed for the transfer function of a URC line resistively terminated at both ends. Based on this representation, a lumped network with three passive elements only can be used for modeling the performance of URC lines with resistive termination at both ends. This model can be easily implemented for computer-aided analysis of circuits comprising URC lines with resistive termination at both ends.

It is worth mentioning that while the analysis presented here assumes that the URC line is resistively terminated at both ends, the same approach can be used to obtain simple models for URC lines with arbitrary impedance termination once the transfer function of the URC line can be expressed in a form similar to eqn. (1).

\section{REFERENCES}

1. G. Deily, Closed-form solutions for voltage-step response of open and shorted RC lines, IEEE Transactions on Circuits and Systems, Vol. CAS-22, pp. 534-541, 1975.

2. M.S. Ghausi and J.J. Kelly, Introduction to Distributed Parameter Networks with Application to Integrated Circuits, New York: Holt, Rinehart and Winston, 1968.

3. R.J. Antinone and G.W. Brown, The modeling of resistive interconnects for integrated circuits, IEEE Journal of Solid-State Circuits, Vol. SC-18, pp. 200-203, 1983.

4. T. Sakurai, Approximation of wiring delay in MOSFET LSI, IEEE Journal of Solid-State Circuits, Vol. SC-18, pp. 418-426, 1983.

5. G. De Mey, A comment on "The modeling of resistive interconnects for integrated circuits," IEEE Journal of Solid-State Circuits, Vol. SC-19, pp. 542-543, 1984. 
6. J.M. Zurada and T. Liu, Equivalent dominant pole approximation of capacitively loaded VLSI interconnection, IEEE Transactions on Circuits and Systems, Vol. CAS-34, pp. 205-207, 1987.

7. J.M. Zurada and T. Liu, Delay estimate in a capacitively loaded URC line, Proc. IEE, Vol. 134, pt. G, pp. 243-245, 1987.

8. M.R. Kaupp, Waveform degradation in VLSI interconnections, IEEE Journal of Solid State Circuits, Vol. 24, pp. 1150-1153, 1989.

9. M.T. Abuelma'atti, Multipole approximation of capacitively loaded VLSI interconnection, Proc. IEE, Vol. 136, pt. G, pp. 114-116, 1989.

10. M.T. Abuelma'atti, Modelling of resistive interconnects for computer-aided analysis of VLSI circuits, Electronics Letters, Vol. 25, pp. 1734-1735, 1989.

11. M.T. Abuelma'atti, The waveform degradation in VLSI interconnections, IEEE Journal of SolidState Circuits, Vol. 25, pp. 1014-1016, 1990.

12. M.T. Abuelma'atti, Modeling of loaded uniform RC lines for computer-aided analysis, IEEE Journal of Solid-State Circuits, Vol. 26, pp. 1470-1472, 1991.

13. M. Milic and M. Gmitrovic, Computation of time-domain response of URC networks, Electronics Letters, Vol. 12, pp. 327-328, 1976.

14. M.L. Liou, A novel method of evaluating transient response, Proc. IEEE, Vol. 54, pp. 20-23, 1966.

15. L.A. Pipes and L.R. Harvill, Applied Mathematics for Engineers and Physicists, New York: McGraw-Hill, pp. 769-771, 1970. 

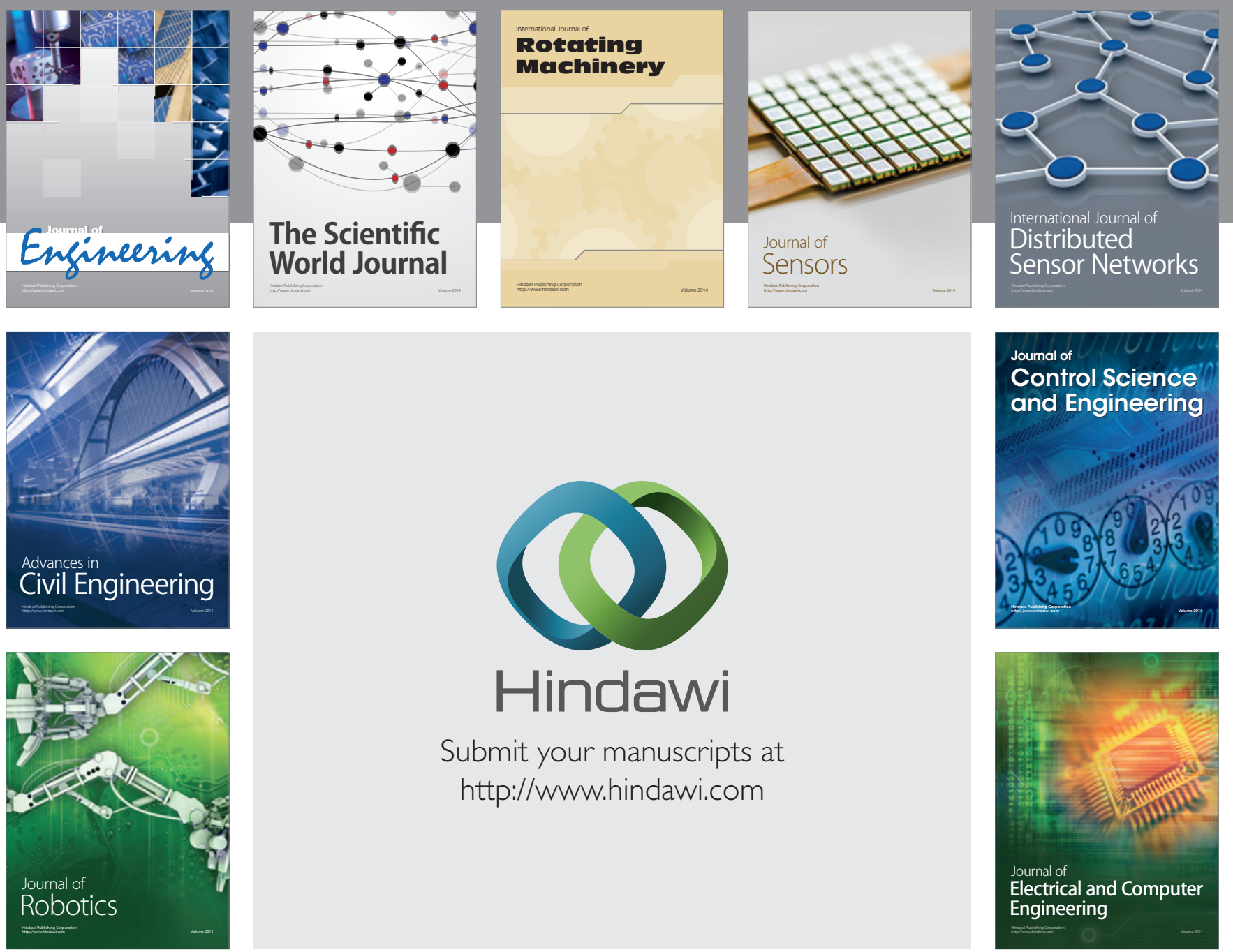

Submit your manuscripts at

http://www.hindawi.com
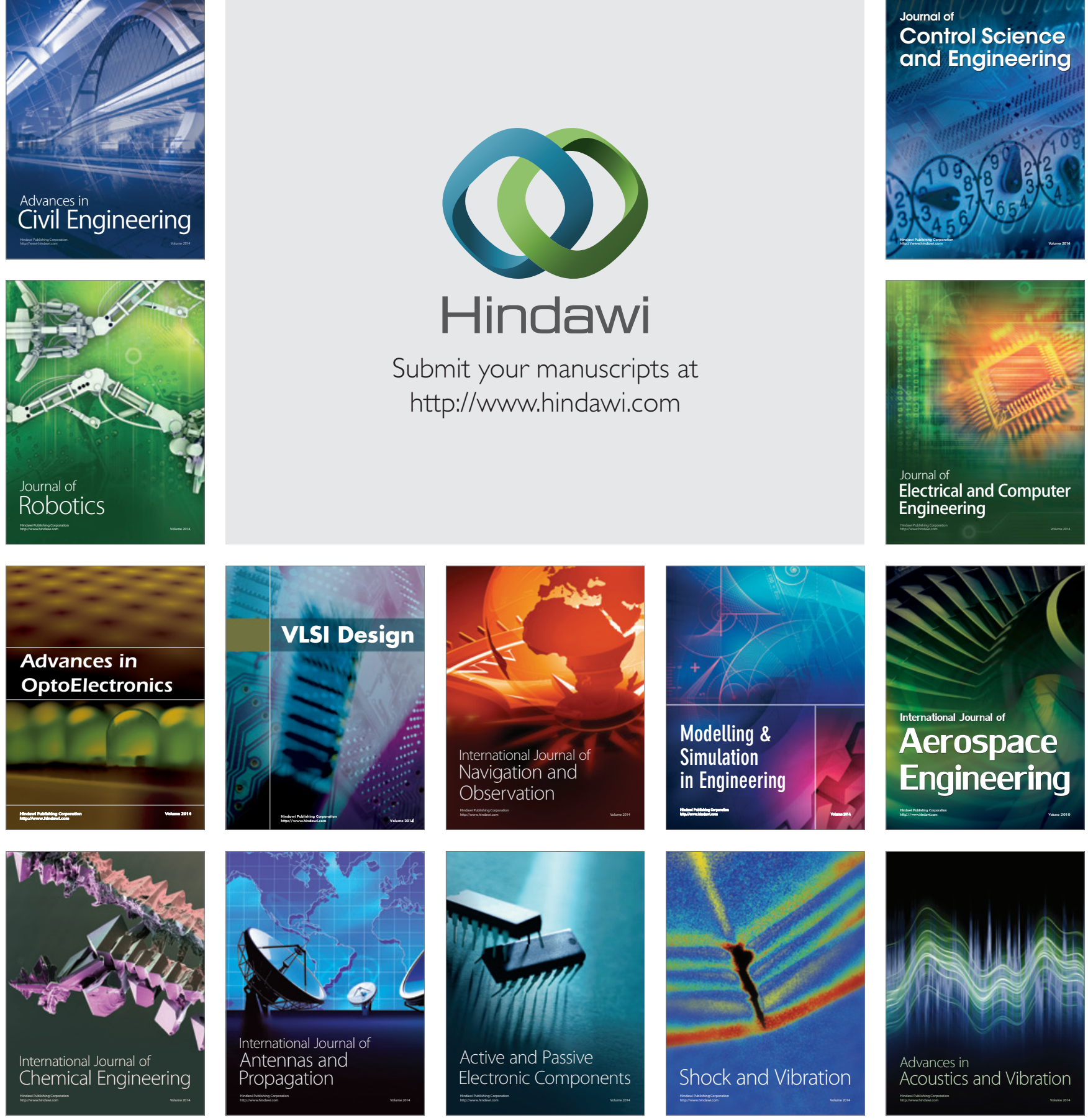